\title{
Is Routine Pregnancy Testing Necessary in Women with Suspected UTI?
}

\author{
B. Navab, I. McAlpine, B. Frazee, J. Maselli, R. Gonzales and J. Stein* \\ University of California San Francisco Emergency Department, 505 Parnassus, Box 0208, San Francisco, CA 94143, USA
}

\begin{abstract}
Objective: To determine if women of reproductive age who present to the Emergency Department (ED) with urinary tract infection (UTI) symptoms can accurately predict their pregnancy status.

Methods: We performed a retrospective study of women who presented to the ED with UTI symptoms (dysuria, frequency, or urgency). Consecutive females aged 18-49 years were referred from triage to a computer kiosk in the waiting room. The kiosk asked all patients in oral and written format: "Are you pregnant, worried you might be pregnant or have you missed your last period?" Patient responses about pregnancy risk were compared to urine pregnancy test results. Test characteristics and post-test probabilities were calculated.

Results: From a total of 300 eligible subjects, 216 received a pregnancy test, of which 10 were positive, giving a pregnancy prevalence of 5\%. Of 196 patients who reported no pregnancy risk, 4 were found to be pregnant, yielding a negative posttest probability of pregnancy $=2.0 \%$ (95\%CI 0.8-5.1). Among the 155 women diagnosed with a UTI, 6 were pregnant, giving a prevalence of $4 \%$. Of the 144 patients who reported no pregnancy risk, 2 were found to be pregnant, yielding a negative posttest probability of $1.4 \%$ (95\% CI 0.4-4.9).

Conclusions: Adult women presenting to the ED with UTI symptoms who report no pregnancy risk have a $2 \%$ or lower probability of actually being pregnant. We believe this level of accuracy to be sufficient for routine clinical practice, and consider it is reasonable to initiate empiric antibiotic therapy without diagnostic pregnancy testing in such patients.
\end{abstract}

Keywords: Urinary tract infection, pregnancy testing.

\section{INTRODUCTION}

\section{Background}

There are approximately 1.4 million emergency department (ED) visits annually by women with urinary tract infection (UTI) in the United States [1]. In the subset of this population that is of reproductive age, it is important to consider pregnancy status because some empiric antibiotic regimens are considered safer than others. A urine pregnancy test is generally recommended in such patients regardless of their menstrual history [2-4]. Implicit in this practice is the perception that self-reported pregnancy status is unreliable.

Several previous studies have evaluated the accuracy of self reported pregnancy status among female patients of reproductive age presenting to the $\mathrm{ED}$, and the results have differed [5-7]. There are no studies that have specifically evaluated women with UTI symptoms.

\section{Significance}

Routine urinalysis may be unnecessary in the diagnosis of UTI for women presenting to the ED with classic symptoms [8]. If patients presenting with UTI symptoms were also found to accurately predict their pregnancy status, it may obviate the need for a urine test of any kind in selected cases. With increasing ED crowding, evaluating such patients without pregnancy testing would be likely to improve the efficiency of care and reduce overall healthcare costs.

*Address correspondence to this author at the University of California San Francisco Emergency Department, 505 Parnassus, Box 0208, San Francisco, CA 94143, USA; Tel: 415-317-1479; E-mail: jstein @ medicine.ucsf.edu

\section{Objective}

We evaluated women who presented to the ED with UTI symptoms by asking three questions regarding their risk for pregnancy. Our hypothesis was that reproductive age women seeking care for urinary symptoms can accurately predict their pregnancy status.

\section{METHODS}

\section{Study Design and Setting}

We performed a retrospective analysis of clinical and laboratory data obtained from a randomized trial of computer-assisted UTI care in female ED patients. Based on commonly utilized UTI telephone triage algorithms, an interactive touch-screen module was developed for the computer kiosk $[9,10]$. In the randomized trial, female patients between the ages of 18 to 65 years of age were referred by the triage nurse to a waiting-room computer kiosk if they presented with symptoms suggesting a UTI. Patients would answer simple questions regarding their symptoms, and if they met criteria for a simple UTI, they were randomized to either standard care (wait until it was their turn to be evaluated in the ED), or rapid care (meet the next available clinician in a triage room for medical screening, and discharged without any further urine or pregnancy testing).

The study was performed at two EDs: 1) University of California San Francisco, an urban tertiary care university hospital with annual census approximately 40,000, and 2) Alameda County Medical Center, an urban county hospital with an annual census of approximately 85,000. Enrollment took place between November 2008 and October 2009. In- 
formed consent was obtained in all patients. This study was approved by the Human Subjects Committee of each hospital.

\section{Selection of Participants}

By study protocol, nurses were instructed to refer all patients presenting to triage with at least one symptom of UTI to the kiosk. Such symptoms included dysuria (any discomfort with urination), urinary frequency, or urinary urgency. We excluded subjects outside of reproductive age, (over 49 years of age). We also excluded the population of women who were eligible for the randomized trial because they did not receive pregnancy testing by protocol. We evaluated our study population as a whole, and designed an a priori subgroup of those patients who had a discharge diagnosis of UTI.

\section{Methods of Measurement}

The computerized module used audio and written formats for all questions. All subjects were asked the following pregnancy screening question: "Are you pregnant now, worried you might be pregnant, or missed your last period?" Answering yes was considered a positive self-reported risk for pregnancy.

To determine actual pregnancy status (the gold standard for diagnosis), we used urine beta human chorionic gonadotrophin (HCG) testing performed during the ED visit. (QuickVue One-Step hCG; Quidel Corp, San Diego, California; and Confirms II, IM Isbell Marthé Diagnostics, Inc, Naples, Florida).

\section{Primary Data Analysis}

We created two-by-two contingency tables to calculate test characteristics for the pregnancy-screening question set. Sensitivity, specificity, positive predictive value, negative predictive value, likelihood ratios, and $95 \%$ confidence in- tervals $(95 \% \mathrm{CI})$ were calculated for the overall population as well as for the subgroup analysis. Statistical analyses were performed using STATA 10 (College Station, Texas, USA).

\section{RESULTS}

Of the 446 female patients referred to the computer kiosk, 80 were outside of our age range, and were excluded (Fig. 1). 66 additional patients were excluded because they were eligible for the randomized trial and did not receive any pregnancy testing by protocol. Of the remaining 300 patients, 84 did not receive a pregnancy test during their ED visit, leaving 216 patients for the current analysis.

Overall, 10 of 216 were pregnant, yielding a prevalence of $5 \%(95 \%$ CI $2.5-8.3)$. A total of 20 patients reported being at risk for pregnancy. Of these, $6(30 \%)$ tested positive. Of the 196 patients who reported no pregnancy risk, $4(2 \%)$ tested positive. Thus, the test characteristics for ruling out pregnancy with patient self-report showed sensitivity of $60 \%$, a negative predictive value of $98 \%$, and a negative likelihood ratio of 0.43 .

Among the subgroup of 155 patients with a physician diagnosis of UTI at discharge, the overall prevalence of pregnancy was 3.9 (95\% CI 1.8-8.2) 11 had a self-reported risk for pregnancy, and $4(36 \%)$ tested positive for pregnancy. Of the 144 patients who reported no pregnancy risk, 2 (1\%) tested positive. In this group, the test characteristics for ruling out pregnancy by patient self-report showed sensitivity of $66.7 \%$, negative predictive value of $98.7 \%$, and a negative likelihood ratio of 0.35 .

\section{DISCUSSION}

In our study of female ED patients presenting with urinary tract symptoms, patient self-reported pregnancy status was reliable enough for use routine clinical care. Patients reporting no to our screening question had a post-test probability of pregnancy of $2 \%$ or lower.

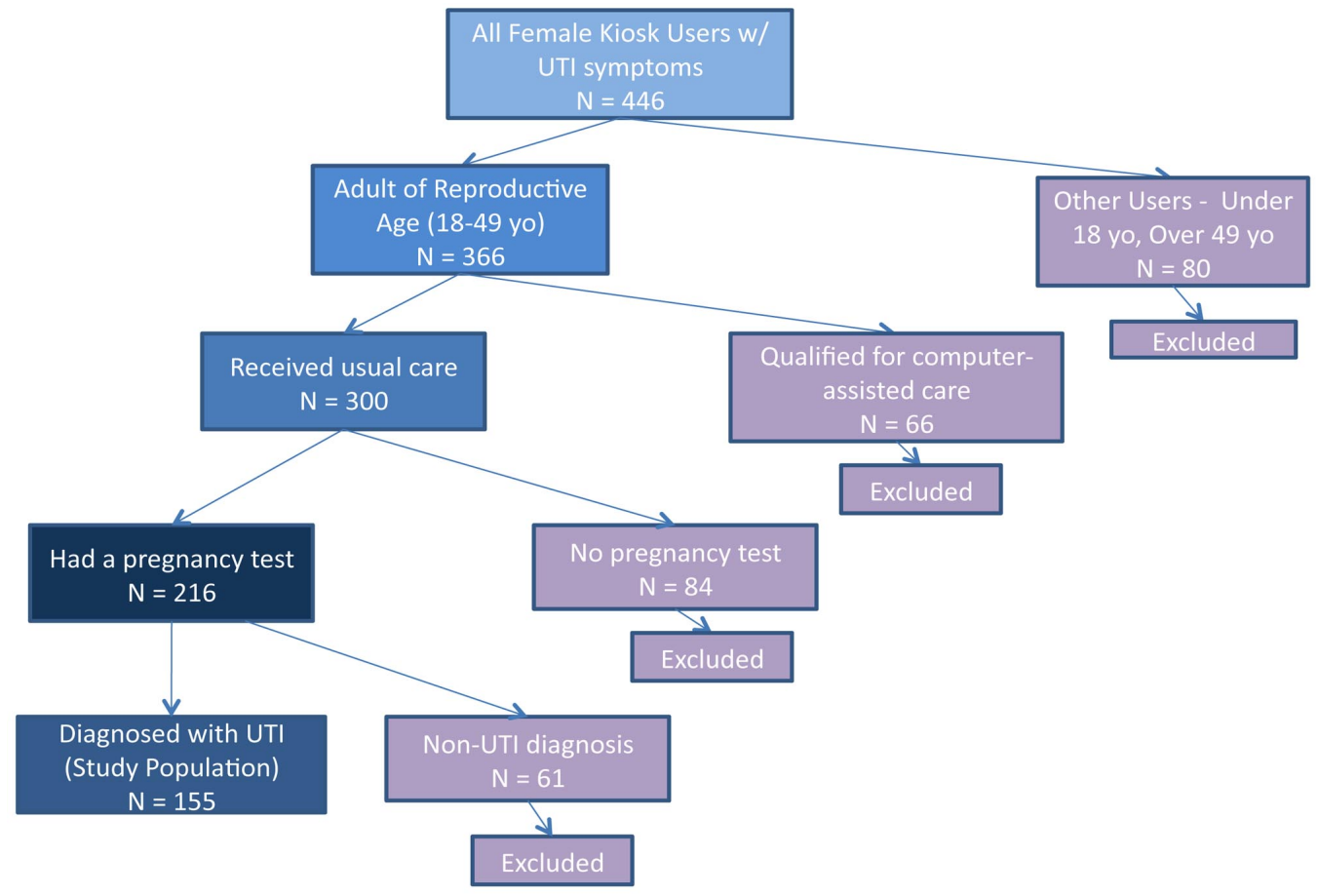

Fig. (1). Study Population. 
In emergency medicine training, a very low threshold for testing pregnancy status in women of reproductive age is recommended. It is common to perform urine pregnancy testing regardless of sexual history, contraceptive use, and menstrual history, because results of pregnancy testing may influence diagnostic testing and treatment decisions [2-4]. Bastian, in JAMA's Rational Clinical Examination series, has proposed that if one can utilize a combination of history or $3 \%$ risk of pregnancy is low enough that testing for pregnancy is unnecessary [11].

We examined patients' self-reported pregnancy risk as a screening diagnostic test that might identify patients with a very low risk of pregnancy who would require no further testing. While sensitivity was modest, the negative predictive value and negative likelihood ratios associated with reporting no risk are sufficiently robust. Given a pregnancy prevalence in our population of $4.6 \%$ overall and $3.9 \%$ in the UTI subgroup, the post-test probability of being pregnant in subjects reporting no risk was $2.0 \%$ and $1.4 \%$, respectively. Thus, by utilizing the simple screening questions, we were able to shift our pregnancy prevalence well below Bastian's $3 \%$ proposed testing threshold.

Three previous studies have examined the reliability of self-reported pregnancy risk among women seeking care in the ED. All were conducted at a single center in an unselected population of female patients [5-7]. The first of these studies, conducted in 1989 , before common use of home pregnancy testing, had an overall pregnancy prevalence of $33 \%$ and found that in subjects reporting no pregnancy risk the prevalence remained $10 \%$ [5]. However, two subsequent studies found that patients reporting no pregnancy risk had pregnancy rates below $2.5 \%$, and both concluded that history was reliable $[6,7]$.

Our study differs from these in that we focused strictly on women presenting to the ED with symptoms consistent with UTI. Reproductive age women with suspected UTI are of particular interest because there is evidence that history alone is often sufficient to diagnose UTI in these patients. In a systematic review, Bent found that typical voiding symptoms and absence of vaginal discharge results in approximately $90 \%$ probability of UTI and that urine testing for diagnostic purposes is unnecessary [8]. In light of this evidence, providers examining reproductive age women with suspected UTI may currently request urine specimens for the sole purpose of pregnancy testing. Our results show that the majority of patients may be sufficiently reliable in determining their pregnancy risk, and the routine practice of urine pregnancy testing may be unnecessary. In the era of ED crowding, changes in practice that allow for the quality of care to be maintained while increasing efficiency are likely to yield benefits to patients as well as delivery systems.

\section{LIMITATIONS}

There were a small number of pregnant women $(n=20)$, resulting in wide confidence intervals around our point esti- mates. In addition, the study was conducted in two urban EDs. In other settings, women with UTI symptoms might be less accurate in predicting their pregnancy status or have a different prevalence of pregnancy which would impact the negative predictive value.

Selection bias could have occurred as a result of triage nurse referral to the computer module. We assume the 84 women who did not receive a pregnancy test were believed to present no risk for pregnancy by the treating provider, however this could also be due to selection bias.

\section{CONCLUSIONS}

In this brief investigation, women of reproductive age who present with urinary tract symptoms and report no risk for pregnancy have a $2 \%$ or lower probability of being pregnant. Our results suggest that urine pregnancy testing may be unnecessary in this population.

\section{ACKNOWLEDGEMENT}

None.

\section{CONFLICT OF INTEREST}

None.

\section{REFERENCES}

[1] Schappert SM, Burt CW. Ambulatory care visits to physician offices, hospital outpatient departments, and emergency departments. Vital Health Stat 2006; 13: 1-66.

[2] Hantsch CE. Normal pregnancy. In: Tintinalli JE, Eds. Emergency Medicine: A Comprehensive Study Guide, $6^{\text {th }}$ ed. New York, McGraw-Hill: 2004; 664-70.

[3] Morrison LJ. General approach to the pregnant patient. In: Marx JA, Eds. Rosen's Emergency Medicine: Concepts and Clinical Practice, $5^{\text {th }}$ ed. St. Louis, MO: Mosby; 2002; 2398-415.

[4] Werman H. Pregnancy testing. In: Harwood-Nuss A, Eds. The Clinical Practice of Emergency Medicine, 3rd ed. Philadelpia, PA: Lippincott, Williams \& Wilkins: 2001; 316-8.

[5] Ramoska EA, Sacchetti AD, Nepp M. Reliability of patient history in determining the possibility of pregnancy. Ann Emerg Med 1989; 18: 48-50.

[6] Stengel CL, Seaberg DC, MacLeod BA. Pregnancy in the emergency department: risk factors and prevalence among all women. Ann Emerg Med 1994; 24: 697-700.

[7] Strote J, Chen G. Patient self assessment of pregnancy status in the emergency department. Emerg Med J 2006; 23: 554-7.

[8] Bent S, Nallamothu BK, Simel DL, Fihn SD, Saint S. Does this woman have an acute uncomplicated urinary tract infection? JAMA 2002; 287: 2701-10.

[9] Barry HC, Hickner J, Ebell MH, Ettenhofer T. A randomized controlled trial of telephone management of suspected urinary tract infections in women. J Fam Pract 2001; 50: 589-94.

[10] Vinson DR, Quesenberry CP, Jr. The safety of telephone management of presumed cystitis in women. Arch Intern Med 2004; 164 : 1026-9.

[11] Bastian LA, Piscitelli JT. Is this patient pregnant? Can you reliably rule in or rule out early pregnancy by clinical examination? JAMA 1997; 278: 586-91. 